\title{
Predictive inverse model allocation for constrained over-actuated linear systems *
}

\author{
Junqiang Zhou ${ }^{\mathrm{a}}$, Marcello Canova ${ }^{\mathrm{a}}$, Andrea Serrani ${ }^{\mathrm{b}}$ \\ ${ }^{a}$ Center for Automotive Research, The Ohio State University, Columbus, OH 43212, USA \\ ${ }^{\mathrm{b}}$ Department of Electrical and Computer Engineering, The Ohio State University, Columbus, OH 43210, USA
}

\begin{abstract}
The paper presents a model predictive allocation scheme for constrained over-actuated linear systems, for which input redundancy entails the existence of multiple trajectories in the state space yielding a given reference output. The method relies upon the concept of inverse model allocation, where dynamic allocation of reference state and input trajectories is accomplished within the framework of output regulation while maintaining invariance of the error-zeroing subspace. The study focuses on the design of model predictive allocator to achieve constraint satisfaction and asymptotic evolution of the trajectories to a precomputed steady-state target. In particular, the objective of this study is the analysis of the stability and feasibility properties of the proposed schemes and the characterization of suitable sufficient conditions for stability in geometric terms. In support of the theoretical findings, this study presents an example where the proposed methodology is applied to solve a constrained tracking control problem for the linearized model of the air path system of a turbocharged Diesel engine.
\end{abstract}

Key words: Constrained Systems; Control Allocation; Geometric Methods; Automotive Control.

\section{Introduction}

Control allocation is a standard tool in the design of control systems for plant models possessing a redundant set of control inputs (see Bodson (2002); Johansen and Fossen (2013) and references therein.) A typical formulation involves squaring the plant model by factoring out the null space of the input map, computing the control policy for the ensuing virtual square system, and lifting the control policy to the original higher dimensional input space by means of optimization techniques. Recent contributions have started to address more challenging cases of input-redundant systems that can not be rendered square by projection. The work of Zaccarian (2009) proposes a taxonomy of input redundant systems, in which the above situation was explicitly recognized. The case of injective input maps in over-actuated systems was termed weak input redundancy to distinguish this scenario from the standard case of strong input redundancy, which can be addressed solely by projection. Geometric

\footnotetext{
* The work was supported by NSF under Grant CMMI1234686. Portion of the material in this paper has been presented at the 2015 American Control Conference.

Email addresses: zhou.412@osu.edu (Junqiang Zhou), canova.1@osu.edu (Marcello Canova), serrani.1@osu.edu (Andrea Serrani).
}

Preprint submitted to Automatica characterizations of weak input redundancy have been recently proposed in the context of full-information output regulation (Serrani, 2012; Galeani et al., 2015), and for more general tracking problems in Cristofaro and Galeani (2014). In these works, it was shown that weakly input redundant systems possess a non-unique inverse model, where redundancy yields the existence of a family of state and input trajectories that are compatible with a given output trajectory. This allows for the definition of control allocation policies aimed at shaping the behavior of the regulated system by providing a suitable on-line selection of the steady-state behavior of the closed-loop system. This selection can be accomplished with performance criteria in mind, which possibly include avoidance of constraint violation in both the input and state space, while preserving invariance of the error-zeroing subspace. In particular, in Serrani (2012) a redundant inverse model is parameterized as a controllable system with assigned dynamics, where a remarkable feature is that the allocation problem is decoupled from error regulation, and can be formulated independently as a dynamic optimization problem. Building upon this work, a model-predictive approach was proposed in (Zhou et al., 2013) for the design of the allocation module, in the specific context of control of over-actuated turbocharged internal combustion engines for automotive applications. 
For systems that are subject to constraints, Model Predictive Control (MPC) is one of the few methodologies that are able to address state/input constraints in a systematic fashion (Mayne et al., 2000). Considerable study has been devoted to application of MPC to the tracking problem, and solutions have been developed by resorting to appropriate formulations in the MPC setting of the internal model principle (Pannocchia and Rawlings, 2003; Limon et al., 2008; Maeder et al., 2009; Maeder and Morari, 2010). For systems with redundant inputs, the use of constrained optimization (Bodson and Frost, 2011) and model predictive control has also been explored in the context of control allocation in applications such as aerospace systems (Luo et al., 2007), thermal management (Vermillion et al., 2011), marine and underwater vehicles (Johansen et al., 2005), automotive systems (Zhou et al., 2013), and fault tolerant control (Cristofaro and Johansen, 2014). The concept of dynamic reference management has also been proposed within the framework of reference governors (Bemporad et al., 1997), where the manipulation of external reference signals is accomplished via predictive optimization.

This paper presents a model predictive allocation scheme for constrained weakly input redundant system, based on the concept of inverse model allocation (Serrani, 2012; Zhou et al., 2013). Differently from reference or command governors, an inverse model allocator aims at optimizing the state/input trajectories while maintaining invariance of the tracking error, that is, without modifying the output reference to be tracked. Moreover, with a compensator pre-designed for stabilizing purposes, the model predictive allocator performs as an add-on module, which mainly focuses on selecting optimal reference trajectories from the redundant family of states/inputs and addressing the constraint requirements. In particular, the cost function to be optimized does not incorporate the tracking error, as opposed to conventional MPC tracking control (Pannocchia and Rawlings, 2003). By expanding on the preliminary results in Zhou et al. (2013), the paper offers a detailed analysis of the stability and feasibility of the proposed approach, and a characterization of sufficient geometric conditions for stability. The investigation focuses on optimality and stability of the allocation of the steady-state trajectories, with an eye to incorporating performance requirements on the transient behavior as well. Finally, a case study is presented to illustrate how the proposed methodology may be applied to a constrained tracking control problem for linearized models of turbocharged Diesel engines characterized by redundant actuation in the air path system.

The paper is organized as follows: Section 2 presents the problem statement. In Section 3, the concept of inverse model allocation is recalled, together with the salient aspects of the synthesis of the extended reference model of Serrani (2012). The design of the dynamic allocation module is described in Section 4, whereas stability and feasibility analysis under the assumption of perfect tracking are presented in Section 5. The case study on the control of the air path system of a linearized overactuated Diesel engine model is presented in Section 6 . Conclusions are offered in Section 7, whereas notation and technical details are found in appendix.

\section{Problem Statement}

Consider the following output regulation problem for discrete-time system

$$
\begin{array}{ll}
w(t+1)=A_{e} w(t) & x(t+1)=A x(t)+B u(t)+B_{e} w(t) \\
y(t)=C x(t) & z=D x(t) \\
r(t)=C_{e} w(t) & e=C x(t)-C_{e} w(t)
\end{array}
$$

with time $t \in \mathbb{N}_{+}:=\{0,1, \cdots\}$, plant state $x \in \mathscr{X} \simeq$ $\mathbb{R}^{n}$, exosystem state $w \in \mathscr{W} \simeq \mathbb{R}^{q}$, control input $u \in$ $\mathscr{U} \simeq \mathbb{R}^{m}$, regulated output $y \in \mathscr{Y} \simeq \mathbb{R}^{p}$, reference $r \in$ $\mathbb{R}^{p}$ and auxiliary performance output $z \in \mathscr{Z} \simeq \mathbb{R}^{s}$. State and input trajectories of the plant model are subject to polyhedron constraints of the form

$$
\mathcal{P} \triangleq\{u \in \mathcal{U}, x \in \mathcal{X}, w \in \mathcal{W}\}
$$

where $\mathcal{U} \subset \mathscr{U}, \mathcal{X} \subset \mathscr{X}$ and $\mathcal{W} \subset \mathscr{W}$ are given compact sets. It is required that $\mathcal{W}$ be a forward invariant set for the exosystem. The following assumptions characterize the class of plant and exosystem models:

\section{Assumption 1}

(1) The pair $(A, B)$ is stabilizable.

(2) The matrix $A_{e}$ is semi-simple with $\operatorname{spec} A_{e} \cap \mathbb{C}^{+}=\emptyset$, where $\mathbb{C}^{+}:=\{\lambda \in \mathbb{C}:|\lambda|>1\}$.

(3) The set of invariant zeros of the triple $(C, A, B)$ and the spectrum of $A_{e}$ are disjoint.

(4) The triplet $(C, A, B)$ is right-invertible.

The linear model (1) is assumed to be over-actuated, that is, $\operatorname{dim} y<\operatorname{dim} u$. It is further assumed that the matrix $B$ has full column rank and the matrix $C$ has full row rank. This scenario has been termed weak input redundancy (Zaccarian, 2009). Finally, it assumed that a dynamic state-feedback stabilizer

$$
\begin{aligned}
& x_{c}(t+1)=A_{c} x_{c}(t)+B_{c} u_{c}(t) \\
& y_{c}(t)=C_{c} x_{c}(t)+D_{c} u_{c}(t)
\end{aligned}
$$

with state $x_{c} \in \mathscr{X}_{c}$, input $u_{c} \in \mathscr{U}_{c}$ and output $y_{c} \in \mathscr{Y}_{c}$ has been designed such that the closed-loop matrix

$$
A_{a}:=\left[\begin{array}{cc}
A+B D_{c} & B C_{c} \\
B_{c} & A_{c}
\end{array}\right]
$$

resulting by setting $u_{c}=x, u=y_{c}$ satisfies $\operatorname{spec} A_{a} \subset$ $\mathbb{C}^{-}$, where $\mathbb{C}^{-}:=\{\lambda \in \mathbb{C}:|\lambda|<1\}$. 


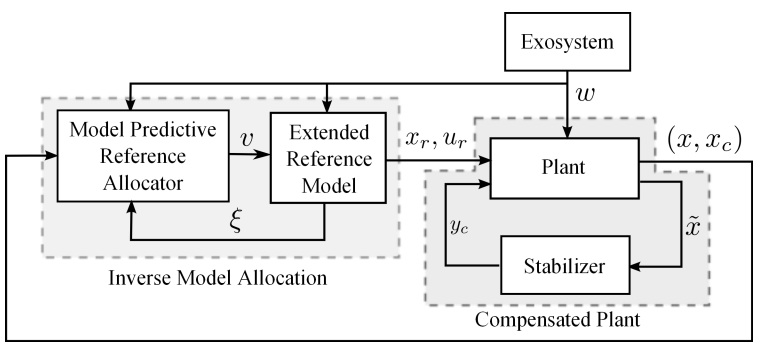

Fig. 1. Architecture of the proposed regulator.

The control goal is to find a full-information plug-in regulator with interconnection structure shown in Figure 1, and the largest possible set of initial conditions such that all trajectories of the closed-loop system remain confined within the constraint set and satisfy $\lim _{t \rightarrow \infty} e(t)=0$, while minimizing a given cost functional $J(z, u)$ of performance output and input trajectories. It is worth noting that the cost function is not required to incorporate penalties on the tracking error. In this study, the cost function is chosen as a quadratic functional of the form

$$
J(z, u)=\sum_{t=0}^{\infty} z(t)^{T} W_{z} z(t)+u(t)^{T} W_{u} u(t)
$$

where $W_{z}, W_{u}$ are positive definite matrices. This is done for convenience, as efficient algorithms are available to calculate the solution (Bemporad et al., 2002).

\section{$3 \quad$ Extended Reference Model Synthesis}

To deal with system constraints in the MPC framework, we adopt a discrete-time formulation of the setup of Serrani (2012), which is briefly recalled for the reader's benefit. As mentioned, the inverse model of weakly inputredundant systems is not unique, hence the steady-state behavior (in the input and state space) can be selected to yield a desired reference output while satisfying additional performance criteria. To this end, Serrani (2012) proposes a method for dynamic allocation of the trajectories of the inverse model of the plant, which is independent from the controller used to steer the closed-loop trajectories to the chosen steady-state behavior. The methodology makes use of an extended reference model, which is a dynamical system of the form

$w(t+1)=A_{e} w(t), \quad \xi(t+1)=\Phi \xi(t)+\Xi v(t)$

$x_{r}(t)=\Pi w(t)+\Sigma \xi(t), u_{r}(t)=\Gamma w(t)+\Psi \xi(t)+\Upsilon v(t)$

with additional state $\xi \in \mathbb{R}^{\rho}$ and input $v \in \mathbb{R}^{m-p}$.

Definition 1 System (5) is said to be an extended reference model for system (1) if for any initial condition $(w(0), \xi(0))=\left(w_{0}, \xi_{0}\right)$ and any $v(\cdot) \in \ell_{\infty}$ the signals $x_{r}(\cdot), u_{r}(\cdot)$ are bounded and satisfy

$$
\begin{aligned}
& x_{r}(t+1)=A x_{r}(t)+B u_{r}(t)+B e w(t), \\
& r(t)=C x_{r}(t)
\end{aligned}
$$

System (5) performs a stable selection (in the $\ell_{\infty}$ sense) of the reference trajectory $\left(x_{r}(\cdot), u_{r}(\cdot)\right)$ that generates the desired output $C_{e} w$ for (1) by means of the assignment of the trajectory $\xi(\cdot)$, which is controlled by the input $v(\cdot)$. Manipulation of $(\xi(\cdot), v(\cdot))$ will be achieved by means of receding-horizon optimization.

The synthesis of the extended reference model in Serrani (2012) will be briefly summarized, as it is instrumental for the results developed in Section 5.2. Standard notation from geometric control is provided in Appendix A.

Proposition 1 Let $(\Pi, \Gamma)$ denote a solution of the regulator equation (Francis, 1977)

$$
\Pi A_{e}=A \Pi+B \Gamma+B_{e}, \quad C_{e}=C \Pi
$$

Let $\mathscr{R}^{\star} \subset \mathscr{X}$ denote the supremal controllability subspace contained in $\operatorname{ker} C$, and let $\mathbb{F}\left(\mathscr{R}^{\star}\right)$ be the set of friends of $\mathscr{R}^{\star}$ (see (Wonham, 1985, Ch.5)). Let ${ }^{1} \rho:=$ $\operatorname{dim} \mathscr{R}^{\star}>0$. Define $\mathscr{V}:=B^{-1} \mathscr{R}^{\star}$. It is known that $\operatorname{dim} \mathscr{V}=m-p$ (Galeani et al., 2015). Select, arbitrarily, a symmetric set $\Omega_{\rho} \subset \mathbb{C}^{-}$and let $F \in \mathbb{F}\left(\mathscr{R}^{\star}\right)$ be such that $\operatorname{spec} A_{F} \mid \mathscr{R}^{\star}=\Omega_{\rho}$, where $A_{F}:=A+B F$. Let $\bar{B}$ denote the domain restriction of $B$ to the subspace $B^{-1} \mathscr{R}^{\star}, \Upsilon$ be the insertion map of $B^{-1} \mathscr{R}^{\star}$ in $\mathscr{U}$ and $\Xi: B^{-1} \mathscr{R}^{\star} \rightarrow \mathscr{R}^{\star}$ be the codomain restriction of the mapping $\bar{B}$ to $\mathscr{R}^{\star}$. Furthermore, let $\Sigma: \mathscr{R}^{\star} \rightarrow \mathscr{X}$ denote the insertion map of $\mathscr{R}^{\star}$ in $\mathscr{X}$, let $\Psi: \mathscr{R}^{\star} \rightarrow \mathscr{U}$ be defined as $\Psi=F \Sigma$ and let $\Phi:=A_{F} \mid \mathscr{R}^{\star}$. With this assignment, system (5) is an extended reference model for (1).

The design of the full-information regulator is accomplished by letting $u_{c}=x-x_{r}$ and $u=y_{c}+u_{r}$. Changing coordinates as $x \mapsto \tilde{x}:=x-x_{r}$ and using the augmented state $\tilde{x}_{a}:=\left(\tilde{x}, x_{c}\right)$ one obtains the following expression of the closed-loop system

$$
\begin{array}{ll}
w(t+1)=A_{e} w(t), & \xi(t+1)=\Phi \xi(t)+\Xi v(t) \\
\tilde{x}_{a}(t+1)=A_{a} \tilde{x}_{a}(t) & e=C_{a} \tilde{x}_{a}
\end{array}
$$

where $C_{a}:=\left[\begin{array}{ll}C & 0\end{array}\right]$. By inspection, system (8) satisfies the control objectives - in particular, asymptotic regulation of $e(t)$-irrespectively of the action of the external input $v(\cdot)$, as long as $v(\cdot) \in \ell_{\infty}$. It is also worth noting that since the augmented plant (with state $\tilde{x}_{a}$ ) is autonomous, its trajectories do not depend on $v(\cdot)$; rather, $\tilde{x}_{a}(t), t \geq 0$, depends uniquely on the initial condition $\tilde{x}_{a}(0)=\left(x(0)-x_{r}(0), x_{c}(0)\right)$, hence on the selection of $\xi(0)$. Therefore, the problem of allocating the reference trajectories is formulated independently from the regulation problem and can be cast as a dynamic optimization problem involving suitable performance criteria and system constraints. Note that if one lets the cost function of the optimization problem depend on $\tilde{x}_{a}(\cdot)$ and

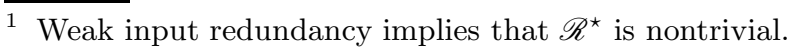


the control $u(\cdot)$, as opposed to depend solely on the references $x_{r}(\cdot)$ and $u_{r}(\cdot)$ as done in Zhou et al. (2013), then stability of the overall interconnection becomes an issue.

\section{Dynamic Allocation Framework}

In this section, a dynamic allocator module is proposed to accomplish the selection of the assignable trajectory $\xi(\cdot)$ via the input $v(\cdot)$, according to the architecture shown in Figure 1. The design of the allocator module aims at selecting the references $x_{r}(\cdot), u_{r}(\cdot)$ so that stability and feasibility properties are enforced in the precompensated system. Consequently, appropriate constraining sets for the references trajectories, $\mathcal{X}_{\epsilon} \subset \operatorname{int} \mathcal{X}$, $\mathcal{U}_{\epsilon} \subset$ int $\mathcal{U}$, are defined such that the constraints $x_{r}(t) \in \mathcal{X}_{\epsilon}, u_{r}(t) \in \mathcal{U}_{\epsilon}$ shall be enforced by the allocator for all $t \geq 0$. This requires the following assumption:

Assumption 2 The set $\mathcal{X}_{\varepsilon}, \mathcal{U}_{\varepsilon}, \mathcal{W}$ are compatible, meaning that there exists a solution $(\Pi, \Gamma)$ of the regulator equation (7) that satisfies $\Pi \mathcal{W} \subset \mathcal{X}_{\epsilon}, \Gamma \mathcal{W} \subset \mathcal{U}_{\epsilon}$.

In addition, we focus on the case of asymptotically constant reference signals, that is, such that for any $w_{0} \in \mathcal{W}$, there exists $w_{\star} \in \mathcal{W}$ such that $\lim _{t \rightarrow \infty} w(t)=w_{\star}$. In this case, the spectrum of $A_{e}$ consists of eigenvalues within the unit circle and simple eigenvalues on the unit circle. Note that the assumption of asymptotically constant references is widely adopted in the MPC literature for its practical relevance and methodological significance alike (Limon et al., 2008; Maeder et al., 2009).

The model predictive paradigm is employed here for the design of the allocation module in Figure 1. Note that M$\mathrm{PC}$ is not used for the design of the stabilizer (3), which is assumed to be given. The strategy adopted for the synthesis of the allocator proceeds as follows: A static optimization procedure (commonly referred to as target calculation) is initially performed to determine an optimal feasible solution in the family of equilibria of the system that are compatible with a given setpoint. Then, a model predictive control allocation policy is derived on the basis of the controllable dynamical system in (5), which shapes the unobservable trajectory of the closedloop system evolving on the subspace $\mathscr{R}^{\star}$ of $\mathscr{X}$.

\subsection{Target Calculation}

Target calculation is a standard procedure when solving tracking problems in the MPC framework (Rao and Rawlings, 1999; Pannocchia and Rawlings, 2003). For a given constant exosystem signal $w(t) \equiv w_{\star}$, an optimal solution shall be determined among all equilibrium pairs $\left(x_{\star}, u_{\star}\right)$ that yields the desired output set-point $C_{e} w_{\star}$. The cost function considered at this stage applies a quadratic penalty to the auxiliary performance output and the actuator efforts, compatibly with the constraints. In particular, the target calculation problem is cast as the solution of the following quadratic program: Given $w_{\star} \in \mathcal{W}$, determine

$$
\begin{gathered}
\left(\xi_{\star}, v_{\star}\right)=\underset{\xi_{\star}, v_{\star}}{\operatorname{argmin}}\left\{z_{\star}^{T} W_{z} z_{\star}+u_{\star}^{T} W_{u} u_{\star}\right\} \\
=\underset{\xi_{\star}, v_{\star}}{\operatorname{argmin}}\left\{x_{\star}^{T} W_{x} x_{\star}+u_{\star}^{T} W_{u} u_{\star}\right\} \\
\text { subject to: } \quad(I-\Phi)^{-1} \xi_{\star}-\Xi v_{\star}=0 \\
x_{\star}=\Pi w_{\star}+\Sigma \xi_{\star} \in \mathcal{X}_{\epsilon}, \quad u_{\star}=\Gamma w_{\star}+\Psi \xi_{\star}+\Upsilon v_{\star} \in \mathcal{U}_{\epsilon}
\end{gathered}
$$

where $W_{z}, W_{u}$ are positive definite matrices, whereas $W_{x}:=D^{T} W_{z} D$ is, in general, positive semi-definite. The existence of admissible solutions of $\left(\xi^{\star}, v^{\star}\right)$ of the problem (9) for any $w_{\star} \in \mathcal{W}$ is guaranteed by Assumption 2.

\subsection{Dynamic Allocation Formulation}

For notational convenience, the dependence on the temporal variable $t$ on signals will be henceforth denoted by a subscript, that is, we let $\zeta_{t}:=\zeta(t)$ denote the value of the signal $\zeta(\cdot)$ at time $t$. Applying the change of coordinates $\tilde{w}:=w-w_{\star}, \tilde{\xi}:=\xi-\xi_{\star}$ and $\tilde{v}:=v-v_{\star}$, the extended reference model (5) is rewritten as the system

$$
\begin{array}{ll}
\tilde{w}_{t+1}=A_{e} \tilde{w}_{t}, & \tilde{\xi}_{t+1}=\Phi \tilde{\xi}_{t}+\Xi \tilde{v}_{t} \\
x_{r_{t}}=x_{\star}+\Pi \tilde{w}_{t}+\Sigma \tilde{\xi}_{t}, & u_{r_{t}}=u_{\star}+\Gamma \tilde{w}_{t}+\Psi \tilde{\xi}_{t}+\Upsilon \tilde{v}_{t}
\end{array}
$$

which has an equilibrium at the origin $(\tilde{w}, \tilde{\xi}, \tilde{v})=$ $(0,0,0)$. Consequently, the deviation from the optimal equilibrium (in the state and input spaces of the augmented plant) reads as

$$
\begin{aligned}
& x_{t}-x_{\star}=\tilde{x}_{t}+\Pi \tilde{w}_{t}+\Sigma \tilde{\xi}_{t} \\
& u_{t}-u_{\star}=C_{c} x_{c_{t}}+D_{c} \tilde{x}_{t}+\Gamma \tilde{w}_{t}+\Psi \tilde{\xi}_{t}+\Upsilon \tilde{v}_{t}
\end{aligned}
$$

As long as the control policy $\tilde{v}(\cdot)$ for the controllable subsystem of (10) is such that the trajectory $\tilde{\xi}_{t}$ is defined for all $t \geq 0$, it follows that $\lim _{t \rightarrow \infty}\left(\tilde{x}_{a_{t}}, \tilde{w}_{t}\right)=(0,0)$ independently from the allocation policy (this is a consequence of asymptotic stability of the dynamics $\tilde{x}_{a_{t+1}}=$ $A_{a} \tilde{x}_{a_{t}}$ and $\left.\tilde{w}_{t+1}=A_{e} \tilde{w}_{t}.\right)$ Hence, boundedness of all trajectories and convergence to the optimal equilibrium defined in (11) (that is, $\lim _{t \rightarrow \infty} x_{t}=x_{\star}$ and $\lim _{t \rightarrow \infty} u_{t}=$ $u_{\star}$ ) are guaranteed if the allocation stabilizes the controllable subsystem of $(10)$ so that $\lim _{t \rightarrow \infty}\left(\tilde{\xi}_{t}, \tilde{v}_{t}\right)=(0,0)$.

With the previous discussion at hand, the cost function for the dynamic reference allocator is selected to penalize the deviations from the optimal equilibrium:

$$
\begin{gathered}
\min _{\tilde{\mathbf{v}}_{t}} J\left(\tilde{x}_{a_{t}}, \tilde{w}_{t}, \tilde{\xi}_{t}, \tilde{\mathbf{v}}_{t}\right)=\sum_{k=0}^{\infty}\left\{\left(x_{k \mid t}-x_{\star}\right)^{T} W_{x}\left(x_{k \mid t}-x_{\star}\right)\right. \\
\left.+\left(u_{k \mid t}-u_{\star}\right)^{T} W_{u}\left(u_{k \mid t}-u_{\star}\right)\right\} \\
\text { subject to: } \tilde{\xi}_{k+1 \mid t}=\Phi \tilde{\xi}_{k \mid t}+\Xi \tilde{v}_{k \mid t}, \quad \tilde{w}_{k+1 \mid t}=A_{e} \tilde{w}_{k \mid t}, \\
\tilde{x}_{a_{k+1 \mid t}}=A_{a} \tilde{x}_{a_{k \mid t}}, \quad x_{k \mid t} \in \mathcal{X}, \quad u_{k \mid t} \in \mathcal{U}, \quad \forall k=0,1,2, \ldots
\end{gathered}
$$


where $(\cdot)_{k \mid t}$ denotes prediction at time $t+k$ from sampling time $t$. Applying the control sequence $\tilde{\mathbf{v}}_{t}:=\left\{\tilde{v}_{0 \mid t}, \tilde{v}_{1 \mid t}, \ldots\right\}$ over the predicted horizon and solving the optimization at each sampling time, one obtains the optimal control law

$$
\tilde{v}_{t}=\tilde{v}_{0 \mid t}^{\star}, \quad \forall t \geq 0
$$

\section{Model Predictive Control Allocation}

A practical way to solve the dynamic optimization (12) is to apply a finite horizon receding optimization, imposed with a terminal cost and terminal constraint set. In particular, the cost function (12) is replaced by

$$
\begin{array}{r}
J\left(\tilde{x}_{a_{t}}, \tilde{w}_{t}, \tilde{\xi}_{t}, \tilde{\mathbf{v}}_{t}\right)=\sum_{k=0}^{N-1}\left\{\left(x_{k \mid t}-x_{\star}\right)^{T} W_{x}\left(x_{k \mid t}-x_{\star}\right)\right. \\
\left.+\left(u_{k \mid t}-u_{\star}\right)^{T} W_{u}\left(u_{k \mid t}-u_{\star}\right)\right\}+V_{f}\left(\tilde{\xi}_{N \mid t}\right)
\end{array}
$$

with terminal cost $V_{f}\left(\tilde{\xi}_{N \mid t}\right):=\tilde{\xi}_{N \mid t}^{T} P \tilde{\xi}_{N \mid t}$, where $P$ is a suitably defined positive definite matrix. The terminal constraint set will be introduced in the sequel. Following the definition of dynamic reference allocator given in (Serrani, 2012, Def. 6.1), the control policy (13) must satisfy the following requirements:

a) Nominal Stability $(0-G A S)$ : When $\tilde{x}_{a} \equiv 0$ and $\tilde{w} \equiv 0$, the origin $\tilde{\xi}=0$ of the system

$$
\tilde{\xi}_{t+1}=\Phi \tilde{\xi}_{t}+\Xi \tilde{v}_{0 \mid t}^{\star}, \quad \tilde{\xi}_{0}=\xi_{0}-\xi^{\star}
$$

is an asymptotically stable equilibrium.

b) Converging Input / Converging State (CICS): There exists a set $\mathcal{X}_{\tilde{\xi}}$ such that for all $\tilde{\xi}_{0} \in \mathcal{X}_{\tilde{\xi}}$ and for any converging feasible trajectory $\left(\tilde{x}_{a_{t}}, \tilde{w}_{t}\right), \tilde{\xi}_{t}$ is a converging signal.

The specific context of this work adds the following requirement:

c) Feasibility: For any converging feasible $\left(\tilde{x}_{a_{t}}, \tilde{w}_{t}\right)$, the constraints are satisfied for the closed loop system, namely, $x_{t} \in \mathcal{X}, u_{t} \in \mathcal{U}, \forall t>0$.

Remark 1 In studying nominal stability, since $\tilde{x}_{a} \equiv$ 0 , it is implicitly assumed that the augmented plant is initialized at the same initial condition as the reference trajectory. Therefore, feasibility of the closed loop system follows from feasibility of the reference trajectory.

\subsection{Nominal Stability}

To prove nominal stability, let $\tilde{x}_{a_{t}} \equiv 0$ and $\tilde{w}_{t} \equiv 0$ in (11) to obtain $x-x_{\star}=\Sigma \tilde{\xi}$ and $u-u_{\star}=\Psi \tilde{\xi}+\Upsilon \tilde{v}$. As a result, the receding horizon optimization (14) becomes

$$
\begin{gathered}
\min J_{a}\left(\tilde{\xi}_{t}, \tilde{\mathbf{v}}_{t}\right)=V_{f}\left(\tilde{\xi}_{N \mid t}\right)+\sum_{k=0}^{N-1}\left\{\left(\Sigma \tilde{\xi}_{k \mid t}\right)^{T} W_{x}\left(\Sigma \tilde{\xi}_{k \mid t}\right)\right. \\
\left.+\left(\Psi \tilde{\xi}_{k \mid t}+\Upsilon \tilde{v}_{k \mid t}\right)^{T} W_{u}\left(\Psi \tilde{\xi}_{k \mid t}+\Upsilon \tilde{v}_{k \mid t}\right)\right\} \\
\text { subject to } \quad \tilde{\xi}_{k+1 \mid t}=\Phi \tilde{\xi}_{k \mid t}+\Xi \tilde{v}_{k \mid t}, \quad x_{\star}+\Sigma \tilde{\xi}_{k \mid t} \in \mathcal{X}, \\
u_{\star}+\Psi \tilde{\xi}_{k \mid t}+\Upsilon \tilde{v}_{k \mid t} \in \mathcal{U}, \quad \tilde{\xi}_{N \mid t} \in \mathcal{E}_{a f}\left(\xi_{\star}\right)
\end{gathered}
$$

for all $k=0,1, \ldots, N-1$

where the terminal constraint set $\mathcal{E}_{a f}\left(\xi^{\star}\right)$, centered at $\xi^{\star}$, is imposed to ensure feasibility. In equation (16), $N \in \mathbb{N}$ is the length of the horizon, whereas the input sequence $\tilde{\mathbf{v}}_{t}=\left\{\tilde{v}_{0 \mid t} \cdots \tilde{v}_{N-1 \mid t}\right\}$ is the optimization variable.

The next proposition establishes conditions for nominal stability and feasibility of the steady-state trajectory. Since feasibility and stability of finite-horizon constrained optimal control have been widely studied (Mayne et al., 2000 ), only a sketch of the proof, which borrows from (Borrelli et al., 2014), will be given.

Proposition 2 Consider the system (10) and the control law given by the solution of the optimization problem (16). Let $\Upsilon^{\dagger}$ denote a left inverse of $\Upsilon$, which exists because the map $\Upsilon: \mathscr{V} \rightarrow \mathscr{U}$ is monic. Assume that the pair $\left(D \Sigma, \Phi-\Xi \Upsilon^{\dagger} \Psi\right)$ is detectable. Define $\mathcal{E}_{0}$ as the set of initial states $\tilde{\xi}_{0}$ for which the optimization (16) is feasible, and select:

i) $\mathcal{E}_{\text {af }}$ as the maximal positive invariant set of the system $\tilde{\xi}_{t+1}=\Phi \tilde{\xi}_{t}$ subject to constraints in (16).

ii) $P$ as the positive definite solution of $-P+\Sigma^{T} W_{x} \Sigma+$ $\Psi^{T} W_{u} \Psi+\Phi^{T} P \Phi \preceq 0$, which exists as $\operatorname{spec} \Phi \in \mathbb{C}^{-}$ by assumption.

Then the following results hold:

(a) The problem (16) is persistently feasible (Borrelli et al., 2014).

(b) The origin $\tilde{\xi}=0$ of the controlled subsystem in (10) is asymptotically stable, with domain of attraction that contains $\mathcal{E}_{0}$.

Proof of Proposition 2. Persistent feasibility and monotonicity of the value function follow by standard arguments, see for instance Mayne et al. (2000). Using as Lyapunov function candidate the value function (16) at its optimal value, $V\left(\tilde{\xi}_{t}\right):=J_{a}\left(\tilde{\xi}_{t}, \tilde{\mathbf{v}}_{t}^{\star}\right)$, and invoking LaSalle's Invariance Principle (LaSalle et al., 1986), trajectories are shown to converge to the largest invariant set $\mathcal{M}$ contained in the set $\mathcal{S}:=\{\tilde{\xi}: D \Sigma \tilde{\xi}=0, \Psi \tilde{\xi}+\Upsilon \tilde{v}=0\}$. In particular

$$
\lim _{t \rightarrow \infty} D \Sigma \tilde{\xi}_{t}=0, \quad \lim _{t \rightarrow \infty} \Psi \tilde{\xi}_{t}+\Upsilon \tilde{v}_{t}=0
$$


and, for any initial condition $\tilde{\xi}_{0} \in \mathcal{M}$, the trajectories of system (15) satisfy

$$
\tilde{\xi}_{t+1}=\left(\Phi-\Xi \Upsilon^{\dagger} \Psi\right) \tilde{\xi}_{t}, \quad D \Sigma \tilde{\xi}_{t}=0
$$

which is the zero dynamics of system (15) under the optimal control policy, with respect to the output $\zeta=$ $D \Sigma \tilde{\xi}$. Since the pair $\left(D \Sigma, \Phi-\Xi \Upsilon^{\dagger} \Psi\right)$ is detectable by assumption, convergence of $D \Sigma \tilde{\xi}_{t}$ implies convergence $\tilde{\xi}_{t}$ to zero. Since $\Upsilon$ is monic, one obtains $\lim _{t \rightarrow \infty} \tilde{v}_{t}=0$.

\subsection{A Generalized Condition for Detectability}

A crucial ingredient of Proposition 2 is detectability of the pair $\left(D \Sigma, \Phi-\Xi \Upsilon^{\dagger} \Psi\right)$, a condition that is expressed in terms of the realization of the extended reference model, and that one must check for all left inverses $\Upsilon^{\dagger}$. A more preferable detectability condition would be expressed in terms of the realization of the original plant model, as this latter could be checked a priori. To this end, we resort to the geometric characterization of the state/input redundancy given in Section 3.

Let $\mathscr{U}$ be expressed as $\mathscr{U}=\mathscr{V} \oplus \mathscr{V}^{\dagger}$, where $\mathscr{V}=B^{-1} \mathscr{R}^{\star}$, and $\mathscr{V}^{\dagger}$ denotes an arbitrary complementary subspace of $\mathscr{V}$. Denote $Q: \mathscr{U} \rightarrow \mathscr{U}$ the projection on $\mathscr{V}^{\dagger}$ along $\mathscr{V}$, and consider, alongside the plant model $\mathscr{P}=\{C, A, B\}$, the "squared" plant model $\mathscr{P}_{s q}:=\{C, A, B Q\}$, where the $m-p$ directions in the input space on $\mathscr{V}$ have been erased $^{2}$. A sufficient condition for the detectability condition is formally stated in Proposition 3, whose proof is given in Appendix B.

Proposition 3 Let $F \in \mathbb{F}\left(\mathscr{R}^{\star}\right)$ be chosen such that the map induced on $\mathscr{S} / \mathscr{R}^{\star}$ by $A_{F}$ has all eigenvalues in $\mathbb{C}^{-}$, where $\mathscr{S}:=\mathscr{R}^{\star}+\langle A \mid \operatorname{im} B\rangle$. Define $A_{Q F}:=A+B Q F$. If the subsystem $\left(D\left|\mathscr{R}^{\star}, A_{Q F}\right| \mathscr{R}^{\star}\right)$ is detectable, then the pair $\left(D \Sigma, \Phi-\Xi \Upsilon^{\dagger} \Psi\right)$, obtained from the corresponding synthesis of the extended reference model, is detectable.

\subsection{Converging-input/Converging-state Property}

Consider now the case $\left(\tilde{x}_{a_{t}}, \tilde{w}_{t}\right) \neq 0$, so that the allocator incorporates in the optimization the information on the transient behavior of the augmented plant. Since the trajectories $\left(\tilde{x}_{a_{t}}, \tilde{w}_{t}\right)$ are governed by autonomous convergent dynamics, boundedness of $\tilde{\xi}(\cdot)$ is guaranteed. However, the transient behavior may produce a violation of the constraints, thus feasibility becomes the primary concern in this case. It is worth noting that the stabilizer has not been defined with the objective of constraint satisfaction in mind, hence the only result that can be expected at this stage is the existence of feasible solution from specified sets of initial conditions. The integration

\footnotetext{
2 Note that the "squared" system has still $m$ inputs, but the
} $m-p$ directions on $\mathscr{V}$ have been erased from the input map. in the stabilizer of mechanisms to prevent constraint violation is the subject of future investigation.

To simplify the notation, define $\eta:=\left(\tilde{w}, \tilde{x}_{a}, \tilde{\xi}\right)$; then the closed loop system (8) is written as

$$
\eta_{t+1}=\Theta \eta_{t}+\Delta \tilde{v}_{t}
$$

where $\Theta=\operatorname{diag}\left\{A_{e}, A_{a}, \Phi\right\}$ and $\Delta=\left[\begin{array}{ccc}0 & 0 & \Xi^{\mathrm{T}}\end{array}\right]^{\mathrm{T}}$. It is readily seen that only the modes associated to $\tilde{\xi}$ are controllable from $\tilde{v}$, and that (19) is Lyapunov stable. In this scenario, the optimization problem (12) becomes:

$$
\begin{aligned}
& \min _{\tilde{\mathbf{v}}_{t}} J_{b}\left(\eta_{t}, \tilde{\mathbf{v}}_{t}\right)=\sum_{k=0}^{N-1}\left\{\left(x_{k \mid t}-x_{\star}\right)^{T} W_{x}\left(x_{k \mid t}-x_{\star}\right)\right. \\
& \left.+\left(u_{k \mid t}-u_{\star}\right)^{T} W_{u}\left(u_{k \mid t}-u_{\star}\right)\right\}+\tilde{\xi}_{N \mid t}^{T} P \tilde{\xi}_{N \mid t} \\
& \text { subject to: } \eta_{k+1 \mid t}=\Theta \eta_{k \mid t}+\Delta \tilde{v}_{k \mid t} \\
& \quad x_{k \mid t} \in \mathcal{X}, \quad u_{k \mid t} \in \mathcal{U}, \quad \tilde{\xi}_{N \mid t} \in \mathcal{E}_{b f}\left(\tilde{w}_{N \mid t}, \tilde{x}_{a_{N \mid t}}, \xi_{\star}\right) \\
& \quad \text { for all } k=0,1, \ldots, N-1
\end{aligned}
$$

Note that terminal penalty and terminal constraint set are considered only with respect to $\tilde{\xi}$ in equation (20), since adding any terminal cost or constraint for the uncontrollable modes will produce no effect.

Proposition 4 Consider the closed loop system (19) and the control law given by $(20)$, and let $\mathcal{O}_{\infty}(\Theta)$ denote the maximal positive invariant set for the autonomous system $\eta_{t+1}=\Theta \eta_{t}$ subject to the constraints in (20). Let the assumptions of Proposition 2 hold, and, in addition:

(1) Let the terminal set be chosen as $\mathcal{E}_{b f}\left(\tilde{w}, \tilde{x}_{a}, \xi_{\star}\right) \triangleq$ $\left\{\tilde{\xi} \in \mathbb{R}^{\rho}: \exists\left(\tilde{w}, \tilde{x}_{a}\right) \in \mathscr{W} \oplus \mathscr{X}_{a}\right.$, s.t. $\left.\eta \in \mathcal{O}_{\infty}(\Theta)\right\}$

(2) Suppose that $\eta_{0} \in \mathcal{K}_{N}\left(\mathcal{O}_{\infty}(\Theta)\right)$, where $\mathcal{K}_{N}\left(\mathcal{O}_{\infty}(\Theta)\right)$ denotes the maximal $N$-step stabilizable set, as defined in (Borrelli et al., 2014, Ch. 11).

Then the receding optimization (20) is persistently feasible and $\tilde{\xi}_{t}$ converges to the origin.

Proof of Proposition 4. By Assumption (2) in the statement of the proposition, there exists a control se-

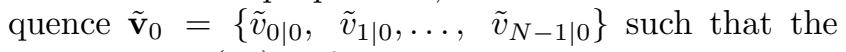
optimization (20) is feasible at time $t=0$. Moreover, combining assumption 1) and assumption 2) it follows that $\mathcal{E}_{b f} \neq \emptyset, \forall t \geq 0$, and that the terminal state $\eta_{N \mid t} \in \mathcal{O}_{\infty}(\Theta)$, which is a control invariant set for the closed loop system (19). As a result, the optimization problem is persistently feasible, (Thm. 13.2, (Borrelli et al., 2014)). To prove stability, recall that the system (19) is asymptotically stable, and that $\tilde{v}=\underline{0}$ is a feasible input in $\mathcal{E}_{b f}$. Therefore, applying $\tilde{v}=\underline{0}$ and by assumption 4) from Proposition 2, the monotonicity of the value 


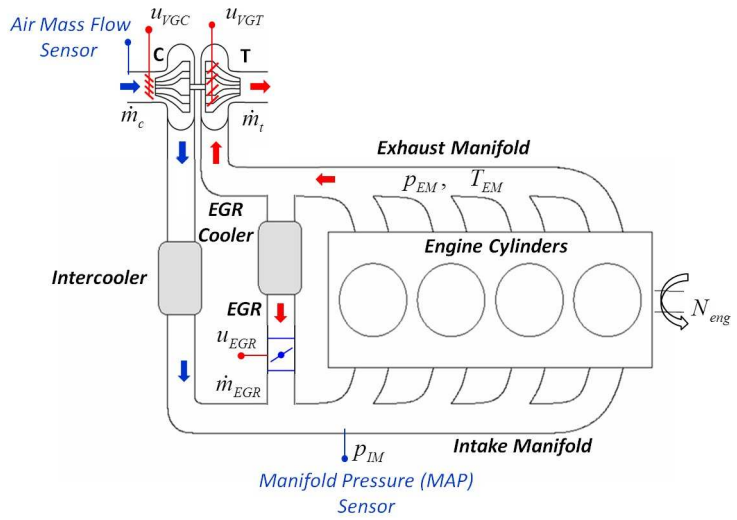

Fig. 2. Scheme of the engine air path system.

function holds for (20). As a result, it follows that

$$
\begin{aligned}
& \lim _{t \rightarrow \infty} D\left(\tilde{x}_{t}+\Pi \tilde{w}_{t}+\Sigma \tilde{\xi}_{t}\right)=0, \\
& \lim _{t \rightarrow \infty} C_{c} x_{c_{t}}+D_{c} \tilde{x}_{t}+\Gamma \tilde{w}_{t}+\Psi \tilde{\xi}_{t}+\Upsilon \tilde{v}_{t}=0
\end{aligned}
$$

Convergence of $\left(\tilde{\xi}_{t}, \tilde{v}_{t}\right)$ follows by convergence of $\left(\tilde{x}_{a}, \tilde{w}\right)$ and the results in Section IV.

Remark 2 Differently from the nominal case, since the initial condition of the plant model reads as $x_{0}=\tilde{x}_{0}+$ $x_{\star}+\Pi \tilde{w}_{0}+\Sigma \tilde{\xi}_{0}$ it is possible to assign the projection of the initial condition $\tilde{x}_{0}$ onto the subspace $\mathscr{R}^{\star}$ via the selection of $\tilde{\xi}_{0}$. In fact, denoting by $\operatorname{Proj}_{\tilde{w}, \tilde{x}_{a}}\left(\mathcal{O}_{\infty}\right)$ the projection of $\mathcal{O}_{\infty}$ onto the subspace $\mathscr{W} \oplus \mathscr{X}_{a}$, and $\Omega_{\tilde{\xi}_{0}}:=$ $\left\{\left(\tilde{w}, \tilde{x}_{a}\right):\left(\tilde{w}, \tilde{x}_{a}, \tilde{\xi}_{0}\right) \in \mathcal{O}_{\infty}\right\}$, it follows that $\Omega_{\tilde{\xi}_{0}} \subset$ $\bigcup_{\tilde{\xi}_{0} \in \operatorname{Proj}_{\tilde{\xi}}\left(\mathcal{O}_{\infty}\right)} \Omega_{\tilde{\xi}_{0}}=\operatorname{Proj}_{\tilde{w}, \tilde{x}_{a}}\left(\mathcal{O}_{\infty}\right)$. Note that $\Omega_{\underline{0}}$ represents the admissible set of $\left(\tilde{w}, \tilde{x}_{a}\right)$ in absence of the extended reference model. Assignability of $\tilde{\xi}_{0}$ allows a possible enlargement of the domain of admissible $\tilde{w}_{0}$ and $\tilde{x}_{a_{0}}$. Thus, the initial feasibility assumption in Proposition 4 may be achieved by an appropriate choice of $\tilde{\xi}_{0}$.

\section{Case Study: Over-actuated Diesel Engine}

To demonstrate the benefits of the proposed regulator design, we present a case study concerning a tracking control problem for air path systems of turbocharged Diesel engines (Guzzella and Amstutz, 1998) characterized by a redundant set of actuators, namely an Exhaust Gas Recirculation (EGR) valve, a Variable Geometry Turbine (VGT), and a Variable Geometry Compressor (VGC) (Canova et al., 2011). A schematic representation of the considered air path system is shown in Figure 2 , illustrating the available actuator set. Production air path systems are typically equipped with air mass flow rate $\dot{m}_{c}$ and intake manifold pressure $p_{I M}$ sensors. Selecting these variables as regulated outputs yields an over-actuated system in the sense of this paper. In tur-

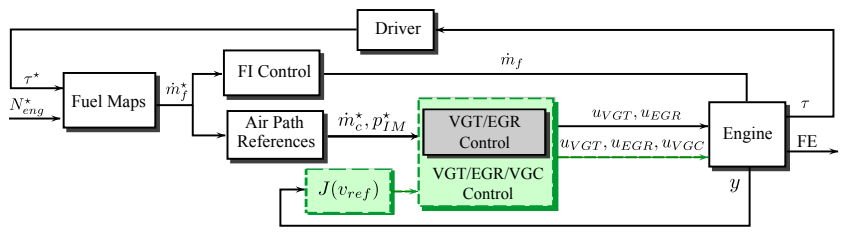

Fig. 3. Proposed air path control systems, as a plugged-in module within a standard EGR-VGT control scheme.

bocharged Diesel engines, the VGT is controlled to alter the amount of energy extracted by the turbine, improving the boost pressure and avoiding over-speeding at high engine speed. The EGR recirculates the exhaust gas into the intake manifold to lower the combustion temperatures and reduce NOx formation. The two actuators are coordinated to achieve a trade-off between fuel economy and emissions, and multivariable tracking controllers are today common practice (Jankovic and Kolmanovsky, 2000; Stefanopoulou et al., 2000). Typically, the objective of the coordinated EGR-VGT control is to regulate $p_{I M}$ and $\dot{m}_{c}$ to suitable set-points $\left(p_{I M}^{\star}, \dot{m}_{c}^{\star}\right)$ obtained from steady-state optimization either experimentally or through the use of high-fidelity engine simulation models. The set-points are scheduled based on the engine speed and torque demand from the driver, and a feedback controller is designed to track the desired targets in transient conditions. However, turbocharged engines are affected by the well-known turbo-lag issue, which has considerable detrimental effects on performance in transient conditions. Downsized engines are further penalized by the risk of operating the compressor close to the surge limit, due to the need of maintaining high boost pressure at low flow rate conditions (i.e., during gear shifts). The use of VGCs have been recently proposed as a solution to directly control the compressor operating point and stability. The additional degree of freedom introduced by the VGC can be exploited to optimize the trajectory of the compressor operating point while steering the output to the desired set-point.

Application of the methodology proposed in this paper aims at the synthesis of an "add-on" controller that can be connected to an existing EGR-VGT controller (see Figure 3.) Due to the considerable calibration effort required to develop a control strategy, redesigning an existing architecture to accommodate the presence of an additional actuator would be a costly and unappealing proposition. Here, dynamic control allocation is used to modify the references for an existing air path system, and does not require modification or recalibration of the tracking controller. Additionally, it relies only on the available set-point $\left(p_{I M}^{\star}, \dot{m}_{c}^{\star}\right)$, as shown in Figure 3; hence the steady-state optimization of the air path system, which involves considerable fine-tuning from engine calibrators, can be preserved in its integrity. Transitions between set-points have been modeled as step responses of stable systems emulating typical engine dynamic$\mathrm{s}$, which can be incorporated in the plant model in the 
Table 1

Engine operating condition for model linearization

$$
\begin{aligned}
& N_{\text {eng }}=1.8 \mathrm{kr} / \mathrm{min}, \dot{m}_{\text {fuel }}=5.53 \mathrm{~g} / \mathrm{s} \\
& p_{I M}=201.7 \mathrm{kPa}, p_{E M}=245.3 \mathrm{kPa}, N_{t c}=79.6 \mathrm{kr} / \mathrm{min} \\
& u_{V G T}=44 \%, u_{E G R}=7 \%, u_{V G C}=50 \% \\
& \dot{m}_{c}=187 \mathrm{~g} / \mathrm{s}, \eta_{c}=69.4 \%
\end{aligned}
$$

form of a suitable exosystem.

\subsection{Air Path System Model and Control Objectives}

In previous work (Taburri et al., 2011), a five-state nonlinear model was developed to capture the low-frequency dynamics of the engine air path system, and validated against experimental data collected on a test engine. A three-state, reduced-order model was then derived to predicts the dynamics of the intake and exhaust manifold pressure (respectively, $p_{I M}$ and $p_{E M}$ ) and the turbocharger speed, $N_{t c}$. Using the notation shown in Figure 2 , the model equations read as:

$$
\begin{aligned}
\frac{d p_{I M}}{d t} & =K_{I M}\left(\dot{m}_{c}+\dot{m}_{E G R}-\dot{m}_{e n g}\right) \\
\frac{d p_{E M}}{d t} & =K_{E M}\left(\dot{m}_{e n g}+\dot{m}_{f u e l}-\dot{m}_{E G R}-\dot{m}_{t}\right) \\
\frac{d N_{t c}}{d t} & =\frac{1}{J N_{t c}}\left(P_{t}-P_{c}\right)
\end{aligned}
$$

where the constants $K_{j}, j=_{I M, E M}$ are computed by averaging the temperature of the fluid (air, exhaust gases) within the intake and exhaust manifold, and $J$ is the rotational inertia of the turbocharger. The nonlinear nature of the model results from the expressions for the mass flow rates $\left(\dot{m}_{c}, \dot{m}_{E G R}, \dot{m}_{e n g}, \dot{m}_{t}\right)$ and the compressor and turbine powers $\left(P_{t}, P_{c}\right)$, which are nonlinear functions of states and inputs of (21). A detailed description of the model is found in Taburri et al. (2011). For control design, the model (21) has been linearized about the engine operating point given in Table 1 .

The exogenous signal, state vector, control input, regulated output and performance output appearing in (1) are respectively given by:

$$
\begin{aligned}
& w=\left[\begin{array}{ll}
N_{\text {eng }} & \dot{m}_{f u e l}
\end{array}\right]^{T}, \quad \quad \quad x=\left[\begin{array}{llll}
p_{I M} & p_{E M} & N_{t c} & u_{V G C}
\end{array}\right]^{T} \\
& u=\left[\begin{array}{lll}
u_{V G T} & u_{E G R} & v_{V G C}
\end{array}\right]^{T} \\
& y=\left[\begin{array}{ll}
p_{I M} & \dot{m}_{c}
\end{array}\right]^{T}, \quad z=\left[\begin{array}{ll}
\eta_{c} & N_{t c}
\end{array}\right]^{T}
\end{aligned}
$$

With a mild abuse of notation, all variables in (22) are now used to represent deviations from the nominal operating condition, whereas in (21) the same notation was employed for the physical variables. Note that the original control input $u_{V G C}$ has been augmented as an additional state with an integrator to remove the direct feedthrough at the output equation by considering the slew rate $v_{V G C}$ as a new input. The auxiliary performance
Table 2

Exosystem model parameters

$$
\omega_{n}=25.12 \mathrm{rad} / \mathrm{s}, \zeta=0.4, \tau=0.707 \mathrm{~s}, \kappa=1.414 \mathrm{~s}
$$
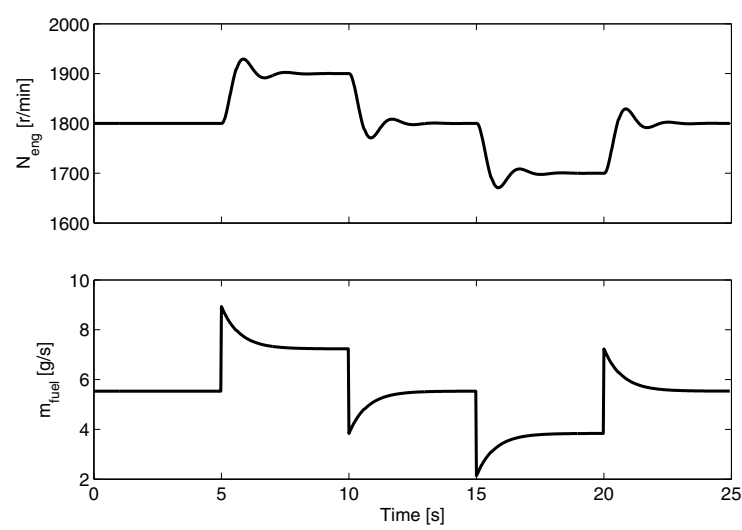

Fig. 4. Exogenous signals: engine speed (top) and fuel flow rate (bottom).

variables $z$ focus primarily on the compressor operating point by considering the turbocharger shaft speed $N_{t c}$ and the compressor efficiency $\eta_{c}$, which can be related to turbo-lag and boosting performance. The constraints for the linearized system (in terms of deviations from nominal operating condition) include saturation limits for the actuators, $u_{V G T} \in[-20,20] \%, u_{E G R} \in[-7,20] \%$, $u_{V G C} \in[-30,30] \%$, and states $p_{I M} \in[-60,60] k P a$, $p_{E M} \in[-75,75] k P a, N_{t c} \in[-30,30] \mathrm{kr} \mathrm{min}^{-1}$, representing permissible deviations from the nominal values. An additional constraint is included to characterize the safe operating region for the compressor, which is limited by surge and choke at low and high flow rate condition$\mathrm{s}$, respectively. The constraint is formulated in terms of the Surge Index $(S I)$, constrained in the range $[5,95] \%$.

The exosystem has been obtained from the discretization of an integrator cascaded with a second-order filter for engine speed, and of an integrator cascaded with a first-order filter for fuel flow rate to generate the timevarying input profiles $\left(N_{\text {eng }}(t), \dot{m}_{f u e l}(t)\right)$ shown in Figure 4 . The parameters of the filters (natural frequency $\omega_{n}$ and damping $\zeta$, and pole time constant $\tau$ and zero time constant $\kappa$, respectively) are given in Table 2 . These values are representative of the engine response during a vehicle tip-in (acceleration) and tip-out (deceleration) transient. The corresponding time-varying reference $r(t)=\left[p_{I M}^{r}(t), \dot{m}_{c}^{r}(t)\right]$ in Figure 6 has been obtained from $\left(N_{\text {eng }}(t), \dot{m}_{\text {fuel }}(t)\right)$ by using a linearization of the aforementioned static feed-forward map.

\subsection{Control Design and Simulation Results}

The control objective is to track the desired set-point of air mass flow rate $\dot{m}_{c}^{\star}$ and intake manifold pressure $p_{I M}^{\star}$, while improving the compressor efficiency $\eta_{c}$ and shaft speed $N_{t c}$ and avoiding compressor surge. These additional performance specifications are to be accomplished 

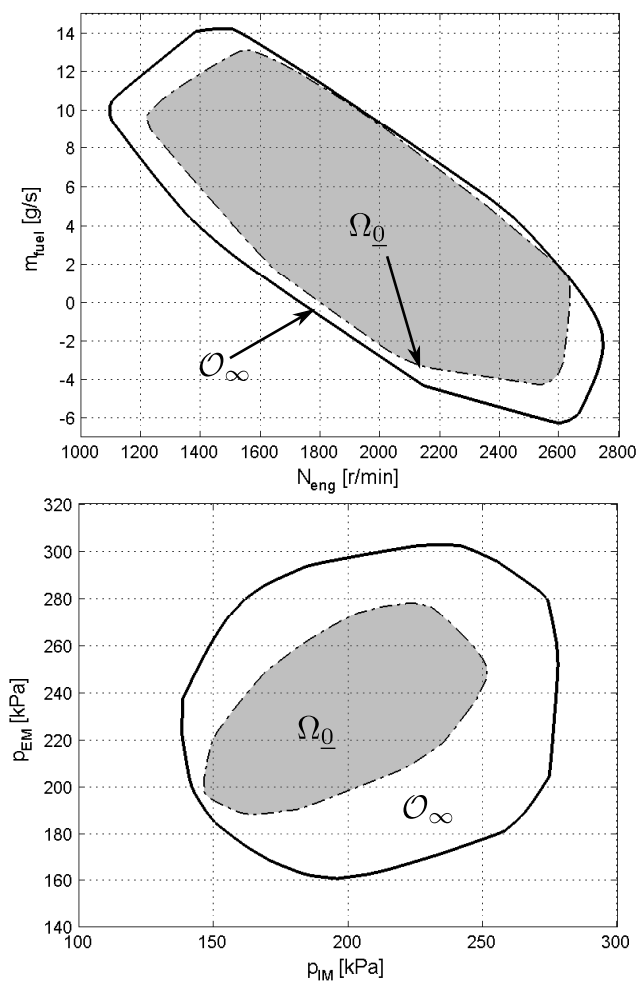

Fig. 5. Maximal feasible set for the exosystem states $N_{\text {eng }}, \dot{m}_{\text {fuel }}$ (top) and plant states $p_{I M}, p_{E M}$ (bottom).

via minimization of the cost functional

$$
J(z, u)=\sum_{t=0}^{\infty} \tilde{z}(t)^{T} W_{z} \tilde{z}(t)+u(t)^{T} W_{u} u(t)
$$

where $\tilde{z}:=z-z_{d}$ denotes deviation from the desired value $z_{d}=[30.6 \% 30.4 \mathrm{kr} / \mathrm{min}]^{\mathrm{T}}$ for $z$, and $W_{z} \in \mathbb{R}^{2 \times 2}$ and $W_{u} \in \mathbb{R}^{2 \times 2}$ are symmetric and positive definite weighting matrices. The extended reference model (5) has state $\xi \in \mathbb{R}^{2}$ and input $v \in \mathbb{R}$. A stabilizer of the form

$x_{c}(t+1)=x_{c}(t)+T_{s} e(t), \quad y_{c}(t)=C_{c} x_{c}(t)+D_{c} u_{c}(t)$

with state $x_{c} \in \mathbb{R}^{2}$ and input $u_{c}(t)=x(t)-x_{r}(t)$, is designed on the basis of the linearized model. The stabilizer incorporates integral actions of the regulated error to achieve offset-free tracking. A standard linear quadratic regulator (LQR) approach has been used to design the stabilizing feedback gain $K_{c}=\left(C_{c} D_{c}\right)$. A sampling time $T_{s}=0.02 s$ and a prediction horizon with $N=5$ are used in this study. Note that robust stability and robust feasibility issues are outside the scope of this paper. Consequently, it has been assumed that the mismatch between the nonlinear and the linearized models is small enough so that stability and feasibility are maintained in the vicinity of the operating condition.

The proposed allocator-based regulator has been tested in simulation on the nonlinear model (21), and com-
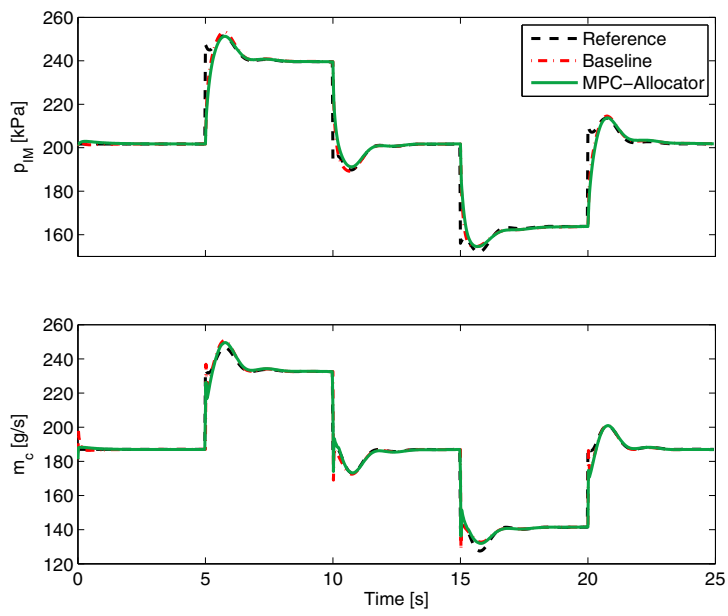

Fig. 6. Regulated output. Dashed line: Reference trajectory; dash-dotted line: Baseline regulator; Solid line: Allocator-based regulator.
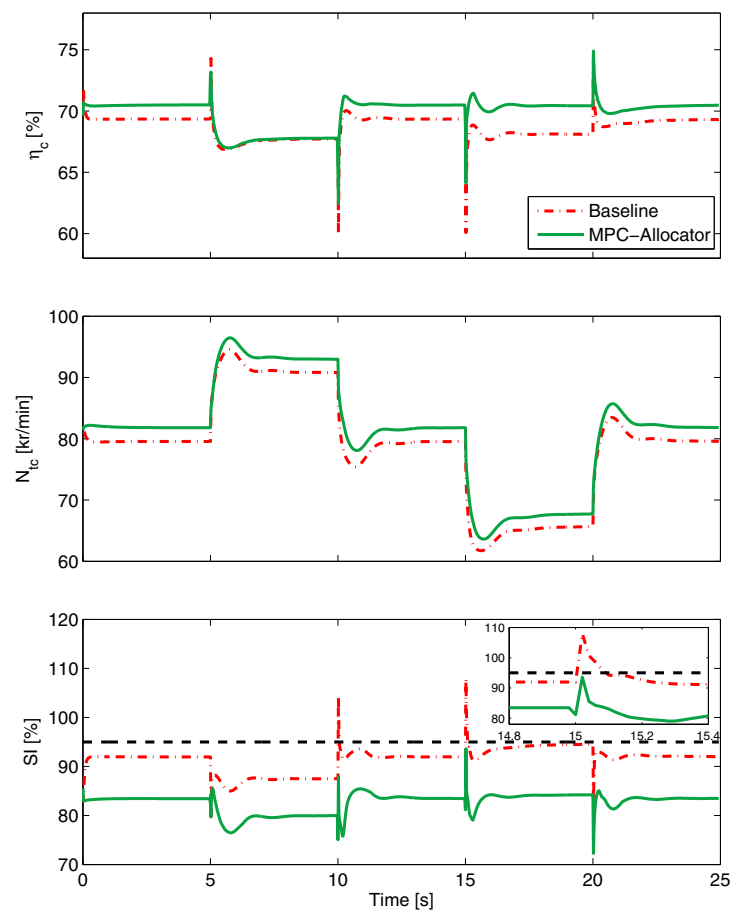

Fig. 7. Auxiliary performance variable. Dashed-dotted line: Baseline regulator; Solid line: Allocator-based regulator.

pared is compared against a baseline regulator obtained by assigning $\xi(0)=0$ and $v(t) \equiv 0$, resulting in the steady state pair $x_{r}(t)=\Pi w(t), u_{r}(t)=\Gamma w(t)$, where $(\Pi, \Gamma)$ solve the regulator equation $(7)$. Note that the $t-$ wo regulators share the same stabilizing feedback gain matrix $K_{c}$. Figure 5 shows the computed maximal feasible sets for the exogenous signals $N_{\text {eng }}, \dot{m}_{f u e l}$ and state $p_{I M}, p_{E M}$ respectively, which represent the projection of $\Omega_{\underline{0}}, \mathcal{O}_{\infty}$ onto the associated subspaces. Note that these 

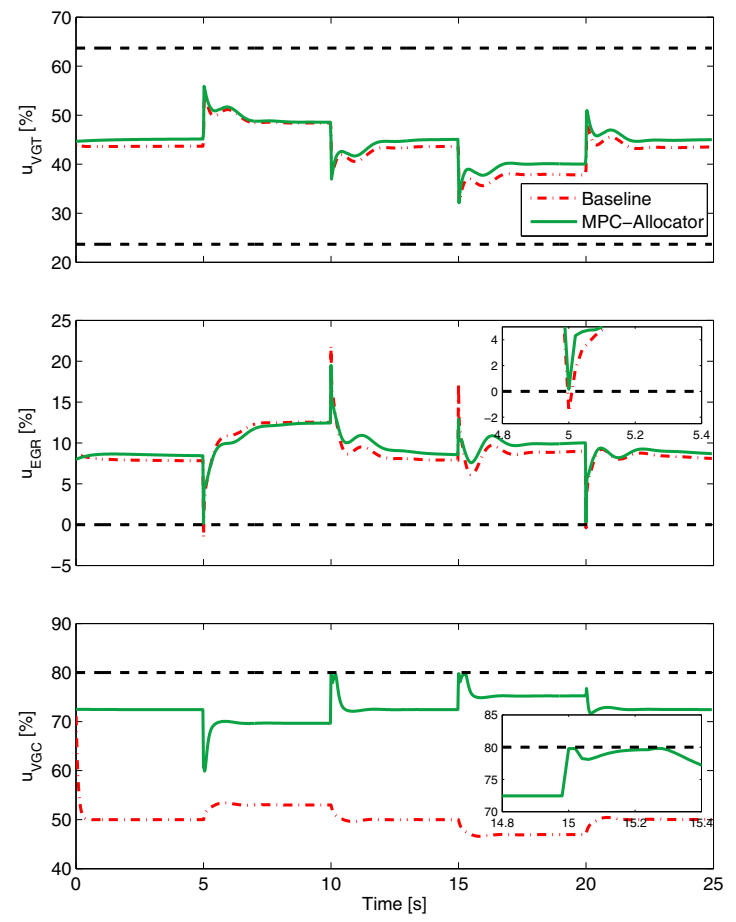

Fig. 8. Time history of the control input. Dash-dotted line: Baseline regulator; Solid line: Allocator-based regulator.

sets are shifted by adding the target values. It is clear that $\Omega_{0} \subset \mathcal{O}_{\infty}$, confirming the advantage (for the linearized model) over a conventional design, as suggested in Remark 2. Figure 6 compares the output reference trajectories and the actual trajectories obtained with the two regulators. The regulated outputs $p_{I M}, \dot{m}_{c}$ converge asymptotically to the reference trajectories, with negligible differences between the two different design approaches. Figure 7 compares the auxiliary performance variables and highlights the improvement achieved by the allocator-based regulator over the baseline. In particular, the proposed approach improves the compressor efficiency and turbocharger shaft speed without affecting the output tracking performance. This result is achieved mainly by a substantial actuation on the VGC, as shown in Figure 8. This outcome can be explained by examining the control authority of the actuators. A simple inspection of the linearized plant model shows that the VGC has the highest control authority on the auxiliary performance variables $\eta_{c}, N_{t c}$ and $S I$, while the EGR and VGT have higher influence on the boost pressure and air flow rate. Although small differences are observed, the VGT and EGR valve positions converge to different steady state values, which is primarily required to compensate the deviations of the regulated variables caused by the VGC actuation. Additionally, the allocator-based regulator is able to maintain the system transient response well within the limits of the inputs, states and performance variables, as opposed to the baseline case. Specifically, the $S I$ is kept within the limit of stability for the compressor at $10 \mathrm{~s}$ and $15 \mathrm{~s}$, as shown in Figure 7, while the VGC and the EGR actuators are saturated at the limits. This is mainly achieved via a proper assignmen$\mathrm{t}$ of the internal dynamics $\xi(t)$ at those instants, which modifies the state $\tilde{x}(t)$ and renders the constrained predicted optimization problem feasible.

\section{Conclusions}

A systematic methodology for the design of optimizing regulators for input-redundant systems has been presented within the framework of inverse model allocation introduced in Serrani (2012). In particular, a modelpredictive approach has been taken in this paper towards the definition of the dynamic allocation module of the regulator architecture proposed therein. The feasibility properties of the ensuing closed-loop system have been investigated, and a sufficient condition for asymptotic stability has been formulated in a coordinate-free setting. A case study on the control of the air path system for a linearized model of a turbocharged Diesel engine has been presented. The results of a nonlinear simulation study confirm the benefits of the proposed methodology over standard regulators, although one should be wary of the local domain of validity due to the linear setup required by the current framework. Efforts are being devoted to extending the applicability of the methodology to linear parameter-varying (LPV) systems and specific classes of nonlinear models of interest.

\section{References}

Bemporad, A., Casavola, A., Mosca, E., 1997. Nonlinear control of constrained linear systems via predictive reference management. IEEE Transactions on Automatic Control 42, 340-349.

Bemporad, A., Morari, M., Dua, V., Pistikopoulos, E.N., 2002. The explicit linear quadratic regulator for constrained systems. Automatica 38, 3-20.

Bodson, M., 2002. Evaluation of optimization methods for control allocation. Journal of Guidance, Control, and Dynamics 25, 703-711.

Bodson, M., Frost, S.A., 2011. Load balancing in control allocation. Journal of Guidance, Control, and Dynamics $34,380-387$.

Borrelli, F., Bemporad, A., Morari, M., 2014. Predictive control for linear and hybrid systems. URL: http://www .mpc.berkeley .edu.

Canova, M., Taburri, M., Fiorentini, L., Chiara, F., Wang, Y.Y., 2011. Modeling and analysis of a turbocharged Diesel engine with variable geometry compressor system. SAE International Journal of Engines 4, 2405-2417.

Cristofaro, A., Galeani, S., 2014. Output invisible control allocation with steady-state input optimization for weakly redundant plants, in: Proceedings of the 53rd IEEE Conference on Decision and Control, Los Angeles, CA. pp. 4246-4253. 
Cristofaro, A., Johansen, T.A., 2014. Fault tolerant control allocation using unknown input observers. Automatica 50, 1891-1897.

Francis, B.A., 1977. The linear multivariable regulator problem. SIAM Journal on Control and Optimization $15,486-505$.

Galeani, S., Serrani, A., Varano, G., Zaccarian, L., 2015. On input allocation-based regulation for linear overactuated systems. Automatica 52, 346-354.

Guzzella, L., Amstutz, A., 1998. Control of Diesel engines. IEEE Control Systems 18, 53-71.

Jankovic, M., Kolmanovsky, I., 2000. Constructive Lyapunov control design for turbocharged Diesel engines. IEEE Transactions on Control Systems Technology 8, 288-299.

Johansen, T.A., Fossen, T.I., 2013. Control allocation: A survey. Automatica 49, 1087-1103.

Johansen, T.A., Fossen, T.I., Tøndel, P., 2005. Efficient optimal constrained control allocation via multiparametric programming. Journal of Guidance, Control, and Dynamics 28, 506-515.

LaSalle, J.P., Hale, J., Meyer, K., 1986. The Stability and Control of Discrete Processes. Springer Verlag, New York, NY.

Limon, D., Alvarado, I., Alamo, T., Camacho, E.F., 2008. MPC for tracking piecewise constant references for constrained linear systems. Automatica 44, 23822387.

Luo, Y., Serrani, A., Yurkovich, S., Oppenheimer, M.W., Doman, D.B., 2007. Model-predictive dynamic control allocation scheme for reentry vehicles. Journal of Guidance, Control, and Dynamics 30, 100-113.

Maeder, U., Borrelli, F., Morari, M., 2009. Linear offsetfree model predictive control. Automatica 45, 22142222 .

Maeder, U., Morari, M., 2010. Offset-free reference tracking with model predictive control. Automatica 46, 1469-1476.

Mayne, D.Q., Rawlings, J.B., Rao, C.V., Scokaert, P., 2000. Constrained model predictive control: Stability and optimality. Automatica 36, 789-814.

Pannocchia, G., Rawlings, J.B., 2003. Disturbance models for offset-free model-predictive control. AIChE Journal 49, 426-437.

Rao, C., Rawlings, J., 1999. Steady states and constraints in model predictive control. AIChE Journal 45, 1266-1278.

Schumacher, J.M., 1980. A complement on pole placement. IEEE Transactions on Automatic Control 25, 281-282.

Serrani, A., 2012. Output regulation for over-actuated linear systems via inverse model allocation, in: Proceedings of the 51st IEEE Conference on Decision and Control, Maui, HI. pp. 4871-4876.

Stefanopoulou, A., Kolmanovsky, I., Freudenberg, J., 2000. Control of variable geometry turbocharged Diesel engines for reduced emissions. IEEE Transactions on Control Systems Technology 8, 733-745.

Taburri, M., Fiorentini, L., Chiara, F., Canova, M.,
Wang, Y., 2011. Modeling and analysis of a turbocharged Diesel engine with variable geometry compressor system. SAE International Journal of Engines 4, 2405-2417.

Vermillion, C., Sun, J., Butts, K., 2011. Predictive control allocation for a thermal management system based on an inner loop reference mode - design, analysis, and experimental results. IEEE Transactions on Control Systems Technology 19, 772-781.

Wonham, W.M., 1985. Linear Multivariable Control. A Geometric Approach. 3rd ed., Springer Verlag, New York, NY.

Zaccarian, L., 2009. Dynamic allocation for input redundant control systems. Automatica 45, 1431-1438.

Zhou, J., Fiorentini, L., Canova, M., Serrani, A., 2013. Dynamic steady-state allocation for over-actuated turbocharged Diesel engines, in: Proceedings of the 52st IEEE Conference on Decision and Control, Firenze, Italy. pp. 6843-6848.

\section{A Terminology and Notation (Wonham, 1985)}

Let $C: \mathscr{X} \rightarrow \mathscr{Y}$ and let $\mathscr{V} \subset \mathscr{X}$ be a subspace with insertion map $V: \mathscr{V} \rightarrow \mathscr{X}$. The domain restriction of $C$ to $\mathscr{V}$ is the map $C \mid \mathscr{V}: \mathscr{V} \rightarrow \mathscr{Y}$ defined by $C \mid \mathscr{V}:=C V$. Let $C: \mathscr{X} \rightarrow \mathscr{Y}$ and let $\mathscr{W} \subset \mathscr{Y}$ be a subspace with insertion map $W: \mathscr{W} \rightarrow \mathscr{Y}$, and $\operatorname{im} C \subset \mathscr{W} \subset \mathscr{Y}$. The codomain restriction of $C$ to $\mathscr{W}$ is the map $\mathscr{W} \mid C: \mathscr{X} \rightarrow \mathscr{W}$ defined by $W(\mathscr{W} \mid C):=C$. If $A \mathscr{V} \subset \mathscr{V}$, the restriction to the invariant subspace $\mathscr{V} \subset \mathscr{X}$ of the endomorphism $A: \mathscr{X} \rightarrow \mathscr{X}$ is the map $A \mid \mathscr{V}: \mathscr{V} \rightarrow \mathscr{V}$ that satisfies $V(A \mid \mathscr{V})=A V$, where $V$ is the insertion map of $\mathscr{V}$ in $\mathscr{X}$. Let $\overline{\mathscr{X}}:=\mathscr{X} / \mathscr{V}$ and denote by $P: \mathscr{X} \rightarrow \overline{\mathscr{X}}$ the canonical projection modulo $\mathscr{V}$. Then there is a unique map $\bar{A}: \mathscr{X} \rightarrow \bar{X}$ such that $\bar{A} P=P A$, where $\bar{A}$ is termed the map induced on $\mathscr{X} / \mathscr{V}$ by $A$. Let $A: \mathscr{X} \rightarrow \mathscr{X}$ and $B: \mathscr{U} \rightarrow \mathscr{X}$. A subspace $\mathscr{V} \subset \mathscr{X}$ is a controlled-invariant subspace of the pair $(A, B)$ if there exists a map $F: \mathscr{X} \rightarrow \mathscr{U}$ such that $(A+B F) \mathscr{V} \subset \mathscr{V}$, where $F$ is termed a friend of $\mathscr{V}$. For a given controlled-invariant subset $\mathscr{V}$ of $\mathscr{X}$, the set of all friends of $\mathscr{V}$ is denoted by $\mathbb{F}(\mathscr{V})$. The controllable subspace associated to the pair $(A, B)$ is the subspace $\langle A \mid \mathscr{B}\rangle:=\mathscr{B}+A \mathscr{B}+\cdots+A^{n-1} B$, where $\mathscr{B}:=\operatorname{im} B$. A subspace $\mathscr{R} \subset \mathscr{X}$ is called a controllability subspace if there exists a map $F: \mathscr{X} \rightarrow \mathscr{U}$ and $G: \mathscr{U} \rightarrow \mathscr{U}$ such that $\mathscr{R}=\langle A+B F \mid \operatorname{im}(B G)\rangle$.

\section{B Proof of Proposition 3}

The proof of Proposition 3 follows by a series of intermediate results established in the form of lemmas.

Lemma 5 The pair $\left(D \Sigma, \Phi-\Xi \Upsilon^{\dagger} \Psi\right)$ is detectable if the pair $\left(D, A+B\left(I-\Upsilon \Upsilon^{\dagger}\right) F\right)$ is detectable.

Proof of Lemma 5. The lemma is proved by contradiction. Assume that the pair $\left(D, A+B\left(I-\Upsilon \Upsilon^{\dagger}\right) F\right)$ is de- 
tectable but the pair $\left(D \Sigma, \Phi-\Xi \Upsilon^{\dagger} \Psi\right)$ is not. Then, there exists $\lambda \in \operatorname{spec}\left(\Phi-\Xi \Upsilon^{\dagger} \Psi\right) \cap \overline{\mathbb{C}}^{+}$and $\xi \in \mathbb{C}^{\rho}, \xi \neq 0$ such that $\left(\Phi-\Xi \Upsilon^{\dagger} \Psi\right) \xi=\lambda \xi$ and $D \Sigma \xi=0$. Since the insertion map $\Sigma: \mathscr{R}^{\star} \rightarrow \mathscr{X}$ is monic, by applying $\Sigma$ on both sides, one obtains $\left(\Sigma \Phi-\Sigma \Xi \Upsilon^{\dagger} \Psi\right) \xi=\lambda \Sigma \xi$. Recalling the synthesis of the extended reference model, the above equation readily implies $(A+B F) \Sigma \xi-B \Upsilon \Upsilon^{\dagger} F \Sigma \xi=\lambda \Sigma \xi$. As a result, there exists $\lambda \in \operatorname{spec}\left(A+B\left(I-\Upsilon \Upsilon^{\dagger}\right) F\right) \cap$ $\overline{\mathbb{C}}^{+}$and $x=\Sigma \xi \in \mathbb{C}^{n}, x \neq 0$ such that

$$
\left(A+B\left(I-\Upsilon \Upsilon^{\dagger}\right) F\right) x=\lambda x, \quad D x=0
$$

which contradicts the assumption.

Lemma 6 The subspace $\mathscr{R}^{\star}$ is a controlled-invariant subspace for $\mathscr{P}_{\text {sq }}$, and each friend of $\mathscr{R}^{\star}$ for $\mathscr{P}$ is also a friend of $\mathscr{R}^{\star}$ for $\mathscr{P}_{s q}$. Morevover, for $\mathscr{P}_{s q}$ the spectrum of the restriction of $A_{Q F}$ to $\mathscr{R}^{\star}, \operatorname{spec}\left(A_{Q F} \mid \mathscr{R}^{\star}\right)$, is fixed for any $F \in \mathbb{F}\left(\mathscr{R}^{\star}\right)$.

Proof of Lemma 6. Let $F \in \mathbb{F}\left(\mathscr{R}^{\star}\right)$. Since $(F-$ $Q F) \mathscr{R}^{\star}=(I-Q) F \mathscr{R}^{\star} \subset \operatorname{im}(I-Q)=B^{-1} \mathscr{R}^{\star}$, then from (Wonham, 1985, Ch.4), it follows that $Q F \in \mathbb{F}\left(\mathscr{R}^{\star}\right)$. Hence, $(A+B Q F) \mathscr{R}^{\star} \subset \mathscr{R}^{\star}$, and the first claim is proven. To prove the second claim, let $F_{i} \in \mathbb{F}\left(\mathscr{R}^{\star}\right), i=1,2$, and $\bar{A}_{Q F i}$ denote the restriction of $A+B Q F_{i}$ to $\mathscr{R}^{\star}$. Again from (Wonham, 1985, Ch.4), it follows that $\left(F_{1}-F_{2}\right) \mathscr{R}^{\star} \subset B^{-1} \mathscr{R}^{\star}$. Then, for any $\bar{x} \in$ $\mathscr{R}^{\star}$, one obtains $\Sigma\left(\bar{A}_{Q F 1}-\bar{A}_{Q F 2}\right) \bar{x}=B Q\left(F_{1}-F_{2}\right) \Sigma \bar{x}$. Since $\Sigma \bar{x} \in \mathscr{R}^{\star}$, it follows that $\left(F_{1}-F_{2}\right) \Sigma \bar{x} \in B^{-1} \mathscr{R}^{\star}$. Recalling that $\operatorname{ker} Q=B^{-1} \mathscr{R}^{\star}$, it follows that $\Sigma\left(\bar{A}_{Q F 1}-\bar{A}_{Q F 2}\right) \bar{x}=0, \forall \bar{x} \in \mathscr{R}^{\star}$. The insertion map $\Sigma$ being monic implies that $\bar{A}_{Q F 1}=\bar{A}_{Q F 2}$, hence the map $A_{Q F} \mid \mathscr{R}^{\star}$, is independent of $F$ for $F \in \mathbb{F}\left(\mathscr{R}^{\star}\right)$.

Fix a basis adapted to the chain $\underline{0} \subset \mathscr{R}^{\star} \subset \mathscr{S} \subset \mathscr{X}$, and a basis adapted to both $\mathscr{V}$ and $\mathscr{V}^{\dagger}$ for $\mathscr{U}$. In these coordinates, the maps $A, B$ and $B Q$ have representation

$$
A=\left[\begin{array}{ccc}
A_{11} & A_{12} & A_{13} \\
A_{21} & A_{22} & A_{23} \\
0 & 0 & A_{33}
\end{array}\right], B=\left[\begin{array}{cc}
B_{11} & B_{12} \\
0 & B_{22} \\
0 & 0
\end{array}\right], B Q=\left[\begin{array}{cc}
0 & B_{12} \\
0 & B_{22} \\
0 & 0
\end{array}\right]
$$

being

$$
\mathscr{R}^{\star}=\operatorname{im}\left[\begin{array}{c}
I_{\rho} \\
0 \\
0
\end{array}\right], \quad \mathscr{V}=\operatorname{im}\left[\begin{array}{c}
I_{m-p} \\
0
\end{array}\right], \quad \mathscr{V}^{\dagger}=\operatorname{im}\left[\begin{array}{c}
0 \\
I_{p}
\end{array}\right]
$$

The fact that $\mathscr{R}^{\star}$ is a controlled-invariant subspace for $\mathscr{P}$ implies that $\operatorname{im} A_{22} \subset \operatorname{im} B_{22}$, hence for any $F \in \mathbb{F}\left(\mathscr{R}^{\star}\right)$

$$
F=\left[\begin{array}{ccc}
F_{11} & * & * \\
F_{21}^{\star} & F_{22} & *
\end{array}\right]
$$

where $*$ denotes unimportant entries, $F_{21}^{\star}$ is such that $B_{22} F_{21}^{\star}=-A_{21}$, and $F_{11}, F_{22}$ assign eigenvalues to the controllable pairs $\left(A_{11}, B_{11}\right)$ and $\left(A_{22}, B_{22}\right)$, respectively. Clearly, for any $F \in \mathbb{F}\left(\mathscr{R}^{\star}\right)$

$$
A+B Q F=\left[\begin{array}{ccc}
A_{11}+B_{12} F_{21}^{\star} & A_{12}+B_{12} F_{22} & * \\
0 & A_{22}+B_{22} F_{22} & * \\
0 & 0 & A_{33}
\end{array}\right]
$$

where $A_{33}$ represents the induced map of $A_{Q F}$ to $\mathscr{X} / \mathscr{S}$, which is fixed for any $F \in \mathbb{F}\left(\mathscr{R}^{\star}\right)$ and $A_{Q F} \mid \mathscr{X} / \mathscr{S}=$ $A \mid \mathscr{X} / \mathscr{S}$. It follows immediately that $(A+B Q F) \mathscr{R}^{\star} \subset$ $\mathscr{R}^{\star}$ for any $F \in \mathbb{F}\left(\mathscr{R}^{\star}\right)$. Also, the second claim implies that the map $A_{11}+B_{12} F_{21}^{\star}$ is fixed for any $F \in \mathbb{F}\left(\mathscr{R}^{\star}\right)$.

Remark 3 For the "squared" triplet $(C, A, B Q)$, the controllability subspace contained in $\operatorname{ker} C$ is $\underline{\underline{O}}$, and $\mathscr{R}^{\star}$ is a controlled-invariant subspace. In particular, $A_{11}+B_{12} F_{21}^{\star}$ is a representation of the induced map $A_{Q F} \mid\left(\mathscr{R}^{\star} / \underline{0}\right)$, which is independent of the choice of $F \in \mathbb{F}\left(\mathscr{R}^{\star}\right)$ and whose spectrum is part of the set of the invariant zeros of $\mathscr{P}_{s q}$ (Schumacher, 1980).

Lemma 7 Let $F \in \mathbb{F}\left(\mathscr{R}^{\star}\right)$ be selected such that $\operatorname{spec}\left(A_{F} \mid \mathscr{S} / \mathscr{R}^{\star}\right) \in \mathbb{C}^{-}$. Denote by $D \mid \mathscr{R}^{\star}$ the domain restriction of the performance output map $D: \mathscr{X} \rightarrow \mathscr{Z}$ to $\mathscr{R}^{\star}$. Then, the pair $\left(D, A+B\left(I-\Upsilon \Upsilon^{\dagger}\right) F\right)$ is detectable if and only if the pair $\left(D\left|\mathscr{R}^{\star}, A_{Q F}\right| \mathscr{R}^{\star}\right)$ is detectable.

Proof of Lemma 7. Since $\Upsilon: \mathscr{V} \rightarrow \mathscr{U}$ is the insertion map from $\mathscr{V}$ to $\mathscr{U}$, the map $\Upsilon \Upsilon^{\dagger}: \mathscr{U} \rightarrow \mathscr{U}$ is the projection on $\mathscr{V}$ along $\mathscr{V}^{\dagger}$. As a result, $Q=I-\Upsilon \Upsilon^{\dagger}$. Denoting by $D=\left[\begin{array}{lll}D_{1} & D_{2} & D_{3}\end{array}\right]$ the representation of $D$ in the basis adapted to $\mathscr{R}^{\star}$ used in the proof of Lemma 6 , detectability of the pair $\left(D, A+B\left(I-\Upsilon \Upsilon^{\dagger}\right) F\right)$ is equivalent to the condition

$$
\begin{aligned}
& \operatorname{rank}\left[\begin{array}{ccc}
A_{11}+B_{12} F_{21}^{\star}-\lambda I & A_{12}+B_{12} F_{22} & * \\
0 & A_{22}+B_{22} F_{22}-\lambda I & * \\
0 & 0 & A_{33}-\lambda I \\
D_{1} & D_{2} & D_{3}
\end{array}\right] \\
& =n+s
\end{aligned}
$$

for all $\lambda \in \operatorname{spec}(A+B Q F) \cap \overline{\mathbb{C}}^{+}$. Since by assumption $\operatorname{spec}\left(A_{22}+B_{22} F_{22}\right)=\operatorname{spec}\left(A_{F} \mid \mathscr{S} / \mathscr{R}^{\star}\right) \subset \mathbb{C}^{-}$, and $\operatorname{spec}\left(A_{33}\right)=\operatorname{spec}(A \mid \mathscr{X} / \mathscr{S}) \subset \mathbb{C}^{-}$by proposition 1 , the above condition becomes

$$
\operatorname{rank}\left[\begin{array}{c}
A_{11}+B_{12} F_{21}^{\star}-\lambda I \\
D_{1}
\end{array}\right]=\rho+s
$$

for all $\lambda \in \operatorname{spec}\left(A_{11}+B_{12} F_{21}^{\star}\right) \cap \overline{\mathbb{C}}^{+}$. The result follows from the fact that $A_{11}+B_{12} F_{21}^{\star}$ and $D_{1}$ are respectively the coordinate representations of $A_{Q F} \mid \mathscr{R}^{\star}$ and $D \mid \mathscr{R}^{\star}$. 